\title{
Constitution and Adventitious Domain Relevance for Establishing Features in Opinion Mining
}

\author{
D. D. Patil ${ }^{1}$, Hafsa N. Mohd Yusuf ${ }^{2}$ \\ ${ }^{1}$ Professor, H.O.D. of Computer Science \& Engineering, S.S.G.B.C.O.E.T., Bhusawal, Maharashtra, India \\ ${ }^{2}$ H.O.D. of Computer Science \& Engineering, S.S.G.B.C.O.E.T., Bhusawal, Maharashtra, India
}

\begin{abstract}
In this paper, we've got an inclination to propose a totally distinctive technique to identify opinion options from on-line reviews by exploiting the excellence in opinion feature statistics across 2 corpora, one domain-specific corpus (i.e., the given review corpus) and one domain-independent corpus (i.e., the contrastive corpus). We have got an inclination to capture this difference via a live called domain affiliation (DR), that characterizes the affiliation of a term to a text assortment. We have got an inclination to initial extract a list of candidate opinion choices from the domain review corpus by method a group of descriptive linguistics dependence rules. For each extracted candidate feature, we've got an inclination to then estimate its intrinsic-domain affiliation (IDR) and extrinsicdomain affiliation (EDR) scores on the domain-dependent and domain-independent corpora, severally. The aim of document-level (sentence-level) opinion mining is to classify the final judgement or sentiment expressed during a personal review document. We, thus, call this interval thresholding the intrinsic and extrinsic domain affiliation (IEDR) criterion. Evaluations conducted on 2 real-world review domains demonstrate the effectiveness of our projected IEDR approach in identifying opinion choices.
\end{abstract}

Keywords: IDR, EDR, IEDR

\section{Introduction}

Opinion mining (also known as sentiment analysis) aimsto analyze people's opinions, sentiments, and attitudestoward entities such as products, services, and their attributes.

Sentiments or opinions expressed in textualreviews are typically analyzed at various resolutions. Forexample, document-level opinion mining identifies theoverall subjectivity or sentiment expressed on an entity(e.g., cellphone or hotel) in a review document, but it doesnot associate opinions with specific aspects (e.g., display,battery) of the entity. This problem also happens, though toa lesser extent, in sentence-level opinion mining.A good many approaches have been proposed toextract opinion features in opinion mining. Supervisedlearning model may be tuned to work well in a givendomain, but the model must be retrained if it is applied to different domains. Unsupervised natural language process (NLP) approaches, determine opinion options by process domain-independent grammar templates or rules that capture the dependence roles and native context of the feature terms. However, rules don't work well on conversational real-life reviews, that lack formal structure. Topic modeling approaches will mine coarse-grained and generic topics or aspects, that are literally linguistics feature clusters or aspects of the particular options commented on expressly in reviews. Existing corpus statistics approaches try and extract opinion options by mining applied mathematics patterns of feature terms solely within the given review corpus, while not considering their spatial arrangement characteristics in another completely different corpus. Our technique is summarized as follows: initial, many grammar dependence rules area unit wont to generate an inventory of candidate options from the given domain review corpus, for instance, radiotelephone or building reviews. Next, for every recognized feature candidate, its domain connectedness score with reference to the domain-specific and domainindependent corpora is computed, that we have a tendency to termed the intrinsicdomain connectedness (IDR) score, and also the extrinsicdomain relevance(EDR) score, severally. within the final step, candidate options with low IDR scores and high EDR scores area unit cropped. We, thus, decision this interval thresholding the intrinsic and external domain connectedness (IEDR) criterion. Evaluations conducted on 2 real-world review domains demonstrate the effectiveness of our projected IEDR approach in characteristic opinion options.

The existing described as: first, several syntactic dependence rules are used to generate a list of candidate features from the given domain review corpus, for example, cellphone or hotel reviews. Next, for each recognized feature candidate, its domain relevance score with respect to the domainspecific and domain independent corpora is computed, which we termed the intrinsic-domain relevance (IDR) score, and the extrinsic domain relevance (EDR) score, respectively. In the final step, candidate features with low IDR scores and high EDR scores are pruned. Thus, call this interval thresholding the intrinsic and extrinsic domain relevance (IEDR) criterion.

\section{Leterature Survey}

In this section we are presented the review of different methods presented for mining high utility item sets from the transactional datasets.

Popescu and O. Etzioni[11].Consumers are often forced to wade through many on-line reviews inorder to make an informed product choice. This paper introduces OPINE, an unsupervised information-extraction system which mines reviews in order to build a model of important product features, their evaluation by reviewers, and their relative quality across products. Compared to previous work, OPINE achieves $22 \%$ higher precision (with only $3 \%$ lower recall) 


\section{International Journal of Science and Research (IJSR) \\ ISSN (Online): 2319-7064}

Index Copernicus Value (2013): 6.14 | Impact Factor (2015): 6.391

on the feature extraction task. OPINE's novel use of relaxation labeling for finding the semantic orientation of words in context leads to strong performance onthe tasks of finding opinion phrases and their polarity.

W. Jin and H.H. Ho.[2].Merchants marketing product on the online typically raise their customers to share their opinions and active experiences on product they need purchased. As e-commerce is changing into additional and additional wellliked, the quantity of client reviews a product receives grows quickly. This makes it tough for a possible client to browse them to create Associate in nursing hip to call on whether or not to buy the merchandise. During this analysis, we tend to aim to mine client reviews of a product and extract extremely specific product connected entities on that reviewers categorical their opinions. Opinion expressions and sentences also known and opinion orientations for every recognized product entity are classified as positive or negative. Completely different from previous approaches that have largely relied on natural language process techniques or datum data, we tend to propose a completely unique machine learning framework exploitation lexicalized HMMs. The approach naturally integrates linguistic options, like part-of-speech and close discourse clues of words into automatic learning. The experimental results demonstrate the effectiveness of the projected approach in internet opinion mining and extraction from product reviews.

N. Jakob and I. Gurevych[3].According to this paper, we have a tendency to concentrate on the opinion target extraction as a part of the opinion mining task. we have a tendency to model the matter as AN info extraction task, that we have a tendency to address supported Conditional Random Fields (CRF). As a baseline we have a tendency to use the supervised formula by Tai et al. (2006), that represents the progressive on the used information. we have a tendency to measure the algorithms comprehensively on datasets from four completely different domains annotated with individual opinion target instances on a sentence level. what is more, we have a tendency to investigate the performance of our CRF-based approach and therefore the baseline during a single- and cross-domain opinion target extraction setting. Our CRF-based approach improves the performance by $0.077,0.126,0.071$ and 0.178 relating to $F$ Measure within the single-domain extraction within the four domains. within the cross-domain setting our approach improves the performance by $0.409,0.242,0.294$ and 0.343 relating to F-Measure over the baseline.

S.-M. Kim and E. Hovy[4], This paper presents a way for characteristic an opinion with its holder and topic, given a sentence from on-line news media texts. we tend to introduce an approach of exploiting the semantic structure of a sentence, anchored to an opinion bearing verb or adjective. This methodology uses participant role labeling as an intermediate step to label an opinion holder and topic victimization information from FrameNet. we tend to decompose our task into 3 phases: characteristic an opinionbearing word, labelling semantic roles associated with the word within the sentence, so finding the holder and therefore the topic of the opinion word among the tagged linguistics roles. For a broader coverage, we tend to additionally use a cluster technique to predict the foremost probable frame for a word that isn't outlined in FrameNet. Our experimental results show that our system performs considerably higher than the baseline.

G. Qiu, B. Liu, J. Bu, and C. Chen[6], Analysis of opinions, called opinion mining or sentiment analysis, has attracted an excellent deal of attention recently because of several sensible applications and difficult analysis issues. during this article, we have a tendency to study 2 necessary issues, namely, opinion lexicon enlargement and opinion target extraction. Opinion targets (targets, for short) square measure entities and their attributes on that opinions are expressed. To perform the tasks, we have a tendency to found that their square measure many grammar relations that link opinion words and targets. These relations may be known employing a dependency programme and so utilised to expand the initial opinion lexicon and to extract targets. This projected methodology relies on bootstrapping. we have a tendency to decision it double propagation because it propagates info between opinion words and targets. A key advantage of the projected methodology is that it solely desires associate degree initial opinion lexicon to start out the bootstrapping method. Thus, the tactic is semisupervised because of the utilization of opinion word seeds. In analysis, we have a tendency to compare the projected methodology with many progressive strategies employing a normal product review check assortment. The results show that our approach outperforms these existing strategies considerably.

\section{Proposed Approach Framework and Design}

\section{A. Problem Defination}

Efficient method for Online Shortest Path Computation on Time Dependent Networks. The previous strategies given for the extraction of opinion feature area unit heavily depending on mining patterns solely from one review corpus, ignoring the nontrivial disparities in word spatial arrangement characteristics of opinion options across totally different corpora. To beat these limitations, recently one methodology introduced for distinctive options in opinion mining via intrinsic and extrinsic domain connection. This methodology is additionally called intrinsic and extrinsic domain connection (IEDR). Much this methodology outperforming existing strategies, however the limitation of this methodology is that it cannot able to extract options non-noun options, rare options, still as implicit options. This becomes new space to enhance during this domain.

Below figure 1 showing the proposed system block diagram and details of algorithm proposed. 


\section{International Journal of Science and Research (IJSR) \\ ISSN (Online): 2319-7064}

Index Copernicus Value (2013): 6.14 | Impact Factor (2015): 6.391

System Architecture

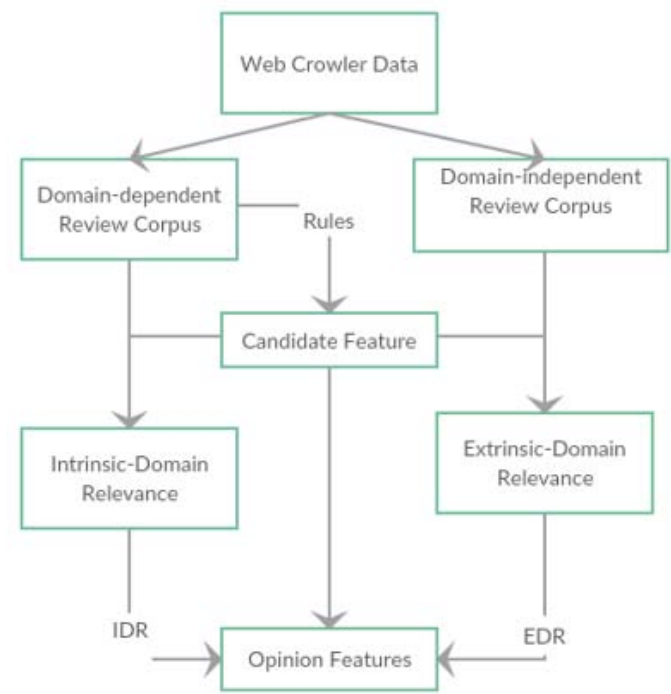

Figure 1: System architecture

In this paper, we have a tendency to propose a completely unique technique to spot opinion features from on-line reviews by exploiting the distinction in opinion feature statistics across two corpora, one domain-specific corpus (i.e., the given review corpus) and one domain-independent corpus (i.e., the contrastive corpus). we have a tendency to capture this inequality via a live known as domain connection (DR), that characterizes the connection of a term to a text assortment. we have a tendency to initial extract a listing of candidate opinion options from the domain review corpus by process a collection of grammar dependence rules. for every extracted candidate feature, we have a tendency to then estimate its intrinsic-domain connection (IDR) and extrinsic-domain connection (EDR) scores on the domaindependent and domain-independent corpora, severally. The aim of document-level (sentence-level) opinion mining is to classify the general judgement or sentiment expressed in a personal review document. We, thus, decision this interval thresholding the intrinsic and adventitious domain connection (IEDR) criterion. Evaluations conducted on two real-world review domains demonstrate the effectiveness of our projected IEDR approach in distinguishing opinion options.

\section{B. Mathematical Model}

Domain relevance characterizes how much a term is related to a particular corpus (i.e., a domain) based on two kinds of statistics, namely, dispersion and deviation.

Both dispersion and deviation are calculated using the wellknown term frequency-inverse document frequency (TFIDF) term weights. Each term Ti has a term frequency TFij in a document $\mathrm{Dj}$, and a global document frequency DFi. The weight wij of term $\mathrm{Ti}$ in document $\mathrm{Dj}$ is then calculated as follows:

$$
w_{i j}=\left\{\begin{array}{c}
\left(1+\log T F_{i j}\right) \times \log \frac{N}{D F_{i}} \text { if } T F_{i j}>0, \\
0 \text { Otherwise, }
\end{array}\right.
$$

where $\mathrm{i} 1 / 41 ; \ldots ; \mathrm{M}$ for a total number of $\mathrm{M}$ terms, and $\mathrm{j} 1 / 4$ $1 ; \ldots ; \mathrm{N}$ for a total number of $\mathrm{N}$ documents in the corpus. The standard variance si for term $\mathrm{Ti}$ is calculated as follows:

$$
s_{i}=\sqrt{\frac{\sum_{j=1}^{N}\left(w_{i j}-\bar{w}_{i}\right)^{2}}{N}}
$$

where the average weight _ wi of term Ti across all documents is calculated by

$$
\bar{w}_{i}=\frac{1}{N} \sum_{j=1}^{N} w_{i j}
$$

The dispersion dispiof each term $\mathrm{Ti}$ in the corpus is defined as follows:

$$
\operatorname{disp}_{i}=\frac{\bar{w}_{i}}{s_{i}}
$$

Dispersion thus measures the normalized average weight of term $\mathrm{Ti}$. It is high for terms that appears frequently across a large number of documents in the entire corpus. The deviation deviijof term $\mathrm{Ti}$ in document $\mathrm{Dj}$ is given by

$$
\operatorname{devi}_{i j}=w_{i j}-\bar{w}_{j}
$$

where the average weight $\mathrm{w}_{\text {_jin }}$ ine document $\mathrm{Dj}$ is calculated over all $\mathrm{M}$ terms as follows:

$$
\bar{w}_{j}=\frac{1}{M} \sum_{i=1}^{M} w_{i j}
$$

Deviation deviijindicates the degree in which the weight wij of the term $\mathrm{Ti}$ deviates from the average $\mathrm{w}_{\text {_jin }}$ the document Dj. The deviation thus characterizes how significantly a term is mentioned in each particular document in the corpus. The domain relevance drifor term $\mathrm{Ti}$ in the corpus is finally defined as follows:

$$
d r_{i}=\operatorname{disp}_{i} \times \sum_{j-0}^{N} d e v i_{i j}
$$

Clearly, the domain relevance driincorporates both horizontal (dispersion dispi) and vertical (deviation deviij) distributional significance of term $\mathrm{Ti}$ in the corpus. The domain relevance score thus reflects the ranking and distributional characteristics of a term in the entire corpus. Note that the domain relevance scores for some terms can be negative, which indicates a relatively weaker association.

\section{Work Done}

In this section, we introduce input dataset, System requirement and practical environment and results.

\section{a)System Specification}

Hardware Requirement:

Processor - Pentium -IV

Speed - $1.1 \mathrm{Ghz}$

RAM - $256 \mathrm{MB}$ (min)

Hard Disk - 20 GB

Key Board - Standard Windows Keyboard

Monitor - SVGA

Software Requirement:

Operating System - Windows XP/7/8/10

Programming Language - Java

Tool - Netbeans. 


\section{International Journal of Science and Research (IJSR) \\ ISSN (Online): 2319-7064}

Index Copernicus Value (2013): 6.14 | Impact Factor (2015): 6.391

\section{b)Results of Practical Work}

Practical work done is as shown in figure given below. Following figure shows the graphical representation of time verses algorithms. Performance is computed according to the time required for set of transactions.

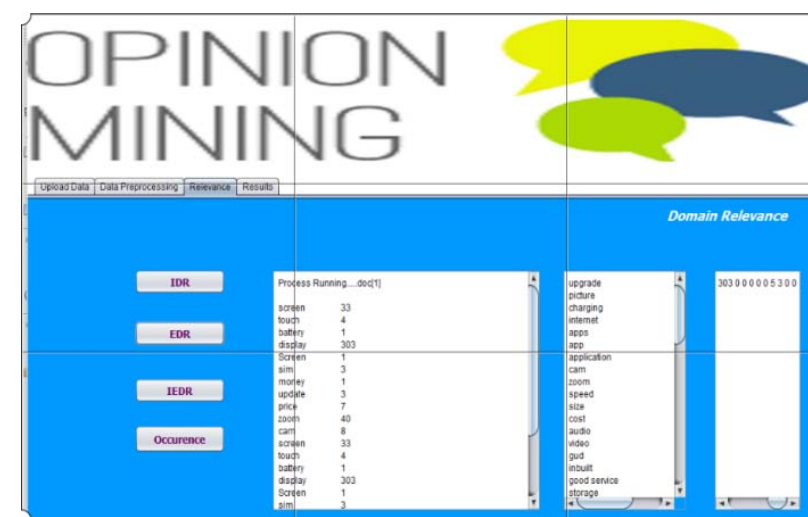

Figure 2: IDER Results

In fig. 2 shows result of our data after applying IDER algorithm.
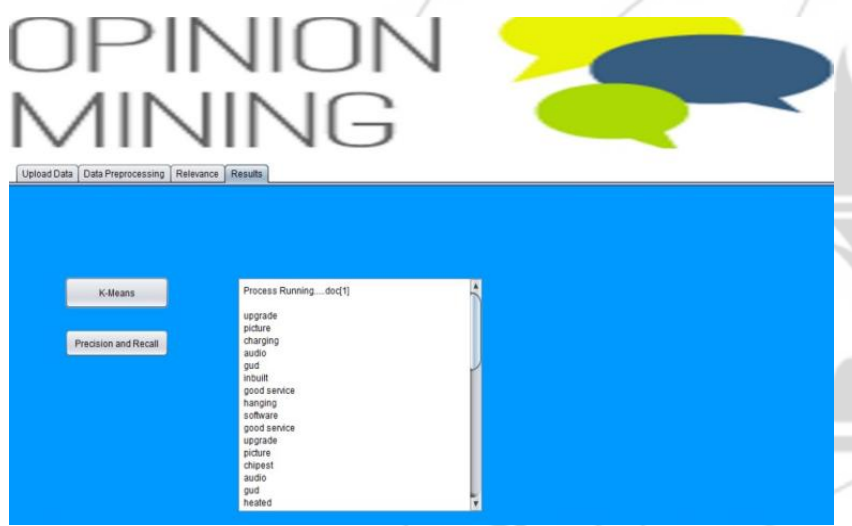

Figure 3: K-means Results

In fig 3. Shows features after applying k-meansalgorithm results for our dataset.

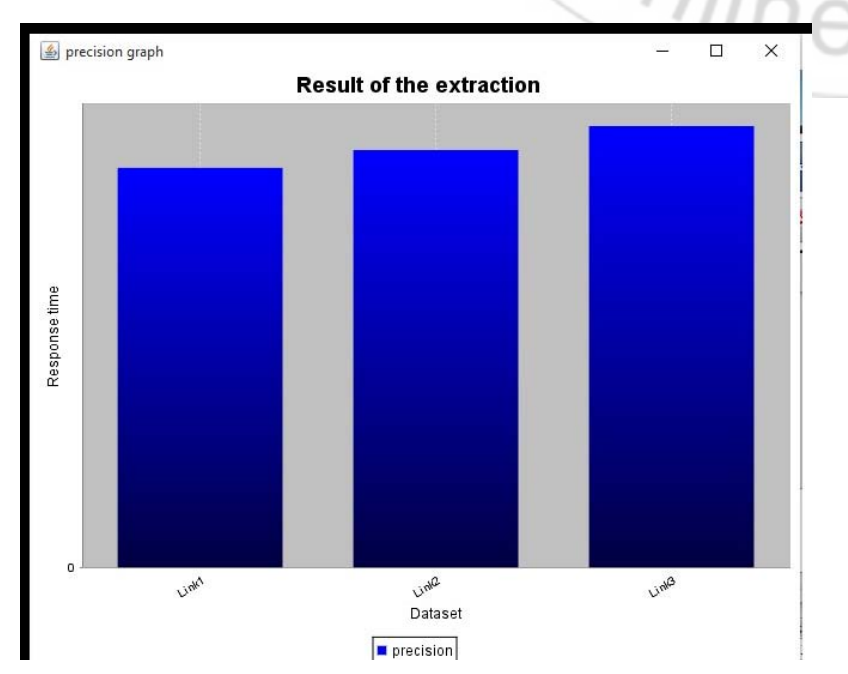

Figure 4: Precision Prediction
In fig 4 we shown prediction of precision for different three links.

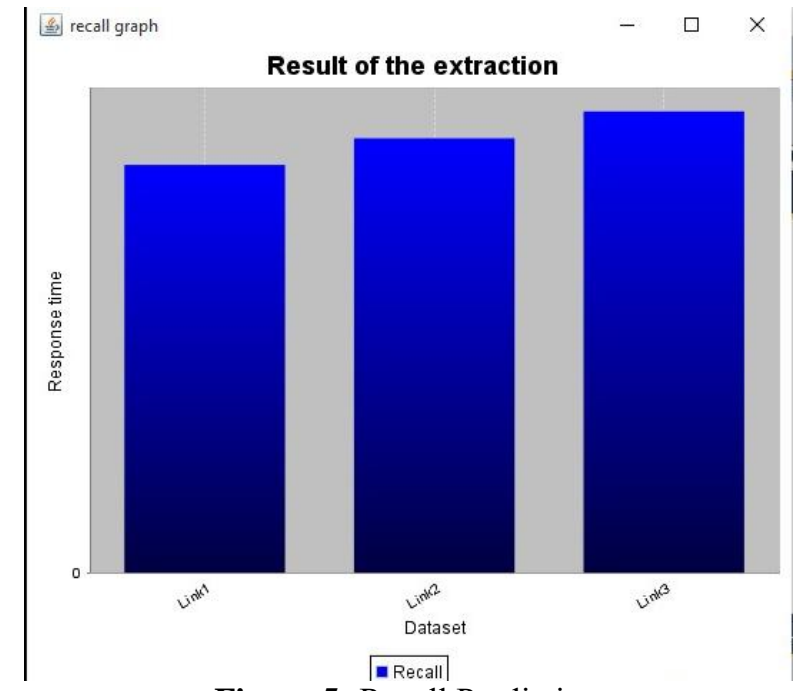

Figure 5: Recall Prediction

In fig 4 we shown prediction of recall for different three links.

\section{Conclusion and Future Work}

In this paper we tend to are implementing new methodology referred to as Hybrid intrinsic and extrinsic domain relevancy (HIEDR) that is predicated on existing IEDR methodology. The goal of this methodology is to extract non solely the options of IEDR however conjointly options like implicit feature, rare options and non-noun options by exploitation fine-grained topic modeling approach. Thus by exploitation Hybrid intrinsic and extrinsic domain relevancy (HIEDR) that is predicated on existing IEDR methodology it provides higher performance. The work done of planned system is shown is result section.

\section{References}

[1] A. Popescu and O. Etzioni, "Extracting Product Features and Opinions from Reviews,"Proc. Human Language Technology Conf. and Conf. Empirical Methods in Natural Language Processing,pp. 339-346, 2005.

[2] W. Jin and H.H. Ho, "A Novel Lexicalized HMMBased Learning Framework for Web Opinion Mining,"Proc. 26th Ann. Int'l Conf. Machine Learning,pp. 465-472, 2009.

[3] N. Jakob and I. Gurevych, "Extracting Opinion Targets in a Singleand Cross-Domain Setting with Conditional Random Fields," Proc. Conf. Empirical Methods in Natural Language Processing, pp. 1035-1045, 2010.

[4] S.-M. Kim and E. Hovy, "Extracting Opinions, Opinion Holders, and Topics Expressed in Online News Media Text," Proc. ACL/COLING Workshop Sentiment and Subjectivity in Text,2006.

[5] G. Qiu, C. Wang, J. Bu, K. Liu, and C. Chen, "Incorporate the Syntactic Knowledge in Opinion Mining in User-Generated Content,"Proc. WWW 2008 Workshop NLP Challenges in the Information Explosion Era,2008.

[6] G. Qiu, B. Liu, J. Bu, and C. Chen, "Opinion Word Expansion and Target Extraction through Double 


\section{International Journal of Science and Research (IJSR) \\ ISSN (Online): 2319-7064}

Index Copernicus Value (2013): 6.14 | Impact Factor (2015): 6.391

Propagation,"Computational Linguistics,vol. 37, pp. 927, 2011.

[7] D.M. Blei, A.Y. Ng, and M.I. Jordan, "Latent Dirichlet Allocation," J. Machine Learning Research,vol. 3, pp. 993-1022, Mar. 2003.

[8] I. Titov and R. McDonald, "Modeling Online Reviews with MultiGrain Topic Models,"Proc. 17th Int'l Conf. World Wide Web, pp. 111-120, 2008.

[9] Y. Jo and A.H. Oh, "Aspect and Sentiment Unification Model for Online Review Analysis,"Proc. Fourth ACM Int'l Conf. Web Search and Data Mining,pp. 815-824, 2011.

[10] M. Hu and B. Liu, "Mining and Summarizing Customer Reviews,"Proc. 10th ACM SIGKDD Int'l Conf. Knowledge Discovery and Data Mining,pp. 168-177, 2004.

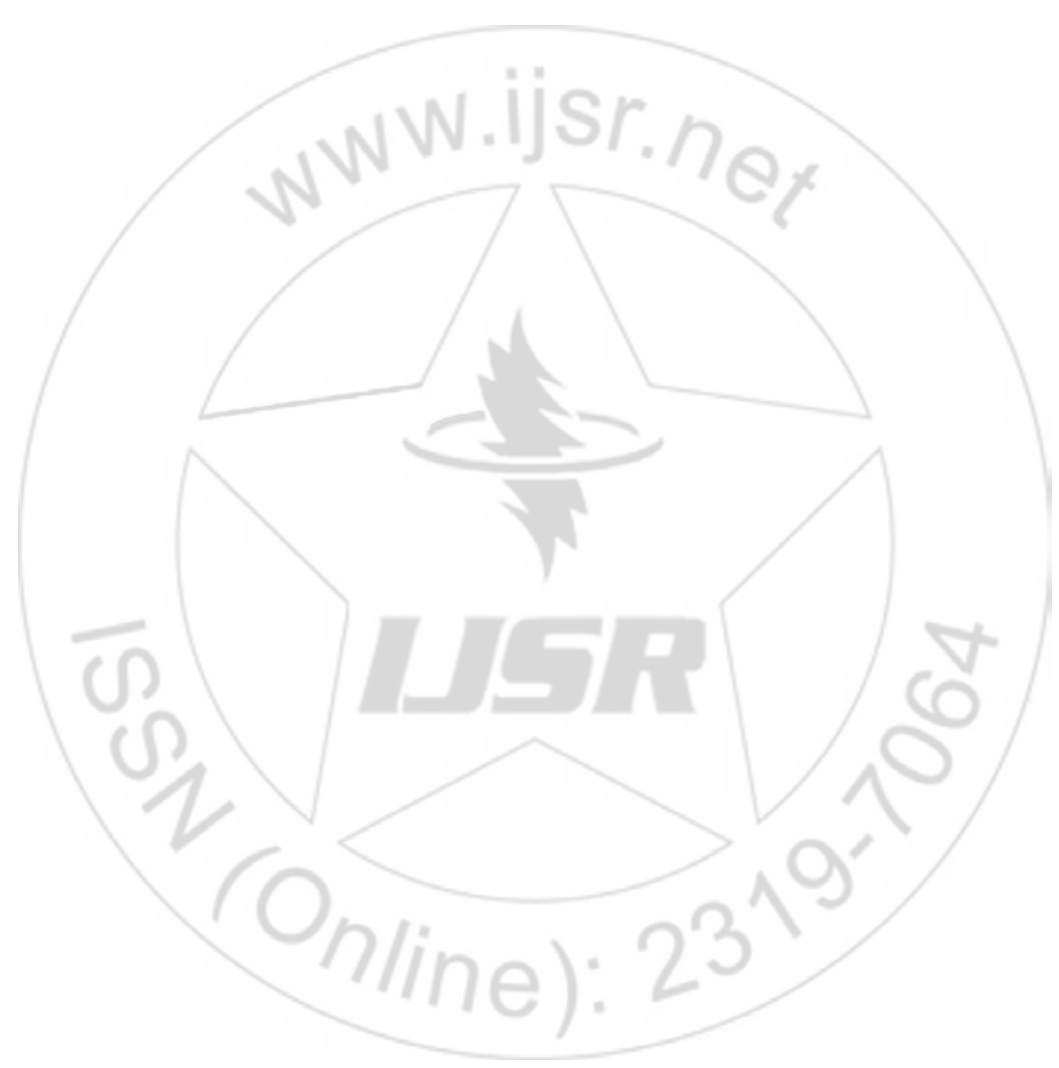

Volume 5 Issue 5, May 2016 